\title{
Association between Anthropometric Parameters and Cardio- Metabolic Disease Risk Factors Among Obese Children and Adolescents
}

\author{
Nayera E Hassan¹, Sahar A El-Masry ${ }^{1}$, Nadia L Soliman², Mona M El-Batran², Muhammad Al-Tohamy ${ }^{1}$, Salwa R El-Batrawy ${ }^{1}$, Mehrevan M \\ Abd El-moniem ${ }^{3}$ \\ ${ }^{1}$ Biological Anthropology Department, Medical Research Division, National Research Centre, El-Bohooth St., Dokki, Giza, Cairo 12622, \\ Egypt; '2Oral and Dental Medicine Dept., Dental Division, National Research Centre, El-Bohooth St., Dokki, Giza, Cairo 12622, Egypt; \\ ${ }^{3}$ Medical Biochemistry Dept., Medical Research Division, National Research Centre, Dokki, Giza, Egypt
}

\begin{abstract}
Citation: Hassan NE, El-Masry SA, Soliman NL, El-Batran MM, Al-Tohamy M, El-Batrawy SR, Elmoniem MMA. Association between Anthropometric Parameters and Cardio-Metabolic Disease Risk Factors Among Obese Children Disease Risk Factors Among Obese Children and Adolescents. Maced J Med Sci. 2012 Jul 31 5(2):152-158. http://dx.doi.org/10.3889 MJMS.1957-5773.2012.0216.

Key words: obesity; anthropometric parameters; fasting blood sugar; lipid profile; cardio metabolic risk factors; children; adolescents.

Correspondence: Prof. Sahar Abd El-Raufe ElMasry. National Research Centre, Biological Anthropology, National Research Centre, ElBohooth St., Dokki, Giza, Cairo 12622, Egypt. Phone:0106606640. E-Mail:asrysa@yahoo.com Received: 03-Oct-2011; Revised: 30-Nov-2011; Accepted: 27-Dec-2011; Online first: 29-May2012

Copyright: (c) 2012 Hassan NE. This is an open access article distributed under the terms of the Creative Commons Attribution License, which permits unrestricted use, distribution, and reproduction in any medium, provided the original author and source are credited.

Competing Interests: The authors have declared that no competing interests exist.
\end{abstract}

\section{Abstract}

Background: Childhood obesity increases risk for developing cardio-metabolic diseases.

Objectives: to investigate the relation of some anthropometric parameters with cardio-metabolic disease risk factors in obese children and adolescents.

Subjects and Methods: Cross sectional study, comprised of 139 obese pupils; aged 8 to 16 years (classified to 3 age groups). Each pupil underwent complete physical examination, anthropometric and laboratory assessment (fasting blood sugar and lipid profile).

Results: Boys had significantly higher values of abdominal skin fold thickness in the age groups II and III and in central adiposity, waist circumference, waist/hip ratio and sub scapular skin fold thickness in adolescents. For boys, fasting blood sugar correlated with most of the anthropometric measurements in young age, and with skinfold thickness in the three age groups. While lipid profile correlated with skinfold thickness particularly at subscapular area in the age groups I and II. For girls, none of the parameters under study showed any significant correlation except subscapular skinfold thickness which had significant correlations with lipid profile in age groups I and II.

Conclusion: Skin fold thickness particularly subscapular; is important indicator for cardio-metabolic complications in obese children of both sexes. Obese boys are more liable to cardio-metabolic complications.

\section{Introduction}

Obesity is a serious global public health problem [1-3]. Concern for children and adolescents, data from NHANEs survey (1976-1980 and 2003-2006) showed that the prevalence of obesity has increased for children aged $6-11$ years from $6.5 \%$ to $17 \%$, and for those aged $12-19$ years, the prevalence has increased from $5 \%$ to $17.6 \%[1,4]$.
It has been widely accepted that obese children and adolescents are at risk for health problems during their youth and adults, i.e. they are more likely to have risk factors associated with cardio-metabolic diseases such as high blood pressure, high cholesterol, and type II diabetes than other healthy, wellbeing children and adolescents [3, 5-8].

Nowadays, obesity is not only of the major causes of life threatening diseases in Western Societies, 
but also, it has become one of the most important health problems in developing countries [9].

In 1998, The World Health Organization (WHO) defined obesity as a condition with excessive fat accumulation in the body, to the extent that health and wellbeing are adversely affected [8].

However, some studies in adults and fewer in children showed the associations between obesity with cardio-metabolic risk factors, such as dyslipidaemia, high blood sugar and high blood pressure [10]. Body mass index (BMI) is usually used to detect obesity although it can't distinguish fat from muscle mass. Waist Circumferences (WC) and waist-to-hip ratio (WHR) are other indices to measure obesity, especially upper abdominal fat distribution (visceral obesity). Despite several publications on the relation between anthropometric markers and lipid profile, the best anthropometric index of fat location remains controversial $[5,11]$.

There is a large body of evidence that obesity related diseases including its cardiovascular consequences begins in childhood [4]. Thus identifying high risk children for targeted interventions is needed. Moreover, there is a large number of questions remain to be answered.

This study focuses to investigate the relation of some anthropometric parameters with cardio-metabolic disease risk factors in obese children and adolescents.

\section{Subjects and Methods}

Data of this cross-sectional study was obtained from 5798 children and adolescents (2655 boys and 3143 girls), aged from 7-18 years, affiliated to 6 public schools in Giza Governorate (2 primary, 2 preparatory and 2 secondary schools) during the period of October, 2007 to April 2009. Permission to perform the study was granted by the "Ethical Committee" of the "National Research Centre", the Ministry of Education, and the directors of the school included in the research. A local consultation with the directors of schools was done for any concerns and ethical issues of the research. Parents were informed about the purpose of the study and their permission in the form of written consent was obtained. Verbal assent was obtained from children and adolescents to be involved in this research.

Of the total sample, those with the complaint of obesity were four hundred and sixty-two students only
(8.0\%); 174 boys and 288 girls; their mean age was $13.43+2.65$ years. Parental informed consent to do lab investigations were obtained only from one hundred and thirty nine pupils (70 boys and 69 girls), with the complaint of obesity. These pupils were required to meet the following inclusion criteria: age, 8-16 years and BMI, greater than the 95th percentile for age and gender based on the Egyptian Growth Reference Charts [12]. These charts group the children every 6 months interval (e.g. 7.25, 7.75, 8.25 years) from 2 years up to 21 years of age. Subjects were excluded from this study for any obvious mentally retarded diseases or those cases suffering from chronic diseases and drug use, or abnormal signs compatible with syndrome obesity. The sample was divided into 3 groups according to their age:

1. group (I): included 27 pupils (16 boys and 11 girls) aged 8-10 years + 6 months;

2. group (II): included 62 pupils (29 boys and 33 girls) aged $11-13$ years + 6 months; and

3. group (III): included 50 pupils ( 25 boys and 25 girls) aged $14-16$ years +6 months.

Each pupil underwent a complete physical examination; including anthropometric measurements (body weight "Wt.", body height "Ht.", waist and hip circumferences and skin fold thickness at triceps, biceps, subscapular, abdominal and suprailiac areas.

Anthropometric measurements: Body height was measured to the nearest $0.1 \mathrm{~cm}$ using a Holtain portable anthropometer. Body weight was determined to the nearest $0.01 \mathrm{~kg}$ using a Seca scale Balance with the subject dressed minimum clothes and no shoes. Waist circumference was measured at the level of the umbilicus with pupil standing and breathing normally, hip circumference at the level of the iliac crest, using nonstretchable plastic tape to the nearest $0.1 \mathrm{~cm}$. Skin fold thickness at the five sites was measured at left side of the body using Harpender Tanner-White house Holtain skin fold caliper to the nearest $0.2 \mathrm{~mm}$. Each measurement was taken; by well-trained researcher and another one assisted to him; as the mean of three consecutive measurements, using standardized equipments and following the recommendations of International Biological Program [13].

The following indices were calculated:

1. Body mass index (BMI): as weight (in kilograms) divided by height (in meters) squared;

2. Waist/ Hip ratio $(\mathrm{cm} / \mathrm{cm})$; 
3. Peripheral obesity: as the sum of triceps and biceps skinfold thickness;

4. Central obesity: as the sum of sub scapular, suprailiac and abdominal skinfold thickness.

Laboratory data: Venous blood samples were obtained to measure plasma glucose level and lipid profile in the morning by venipuncture after after 12hours overnight fasting. Professional staff performed venipuncture. The blood samples were left to clot; sera were separated by centrifugation for 10 minutes at 5000 rpm then stored at $-80^{\circ} \mathrm{C}$ until assays. Plasma glucose was determined by the glucose oxidase method. Plasma concentrations of total cholesterol [14], triglycerides [15], and high-density lipoprotein-cholesterol (HDL-C) [16] were measured using commercially available kits provided by STANBIO Laboratory Inc.(1261 North Main Street Boerne Texas 78006 USA). LDL-C was calculated according to an equation developed by Friedewald et al., [17] as follows:

\section{LDL-C $=$ Total cholesterol - Triglycerides/5+ HDL-C.}

Statistical Analysis: Data was examined for normal distribution using "One-Sample KolmogorovSmirnov Test". All the anthropometric and laboratory parameters were normally distributed $(p<0.05$, and SD $<25 \%$ of the mean value) except waist circumference and waist/hip ratio in age groups II and III ( $p>0.05)$. Statistical evaluation of the results was performed with the SPSS version 9.05 computer programs. All values are reported as the mean $\pm S D$. Sex differences were examined using "Student's $t$ - test "in case of normal distributed data and "Mann-Whitney Test" in case of data not normally distributed. Pearson's correlation coefficients were used to measure the degree of association between anthropometric parameters and lipid profile in both gender and in all age groups in case of normally distributed data, while Spearman' s correlation coefficients were used in case of data not normally distributed. The level of significance was set at a probability of less than $5 \%(p<0.05)$.

\section{Results}

The total sample included 139 pupils, 50.4\% were boys ( $n: 70)$ and 49.6\% were girls (n: 69). Descriptive data for the anthropometric parameters, fasting blood glucose and lipid profile for boys and girls are shown in Tables 1-3. Analyses of the pupils of group (I) aged 8 10 years \pm 6 months (Table 1 ) revealed significant gender differences in some anthropometric
Table 1: Anthropometric characteristics and laboratory data of the group (I) by sex (Age 8-10 \pm 6 months).

\begin{tabular}{|c|c|c|c|c|c|}
\hline \multirow[t]{2}{*}{ Parameters } & \multicolumn{2}{|c|}{$\begin{array}{c}\text { Boys } \\
(N=16)\end{array}$} & \multicolumn{2}{|c|}{$\begin{array}{c}\text { Girls } \\
(N=11)\end{array}$} & \multirow[t]{2}{*}{$p$} \\
\hline & Mean & $+\mathrm{SD}$ & Mean & $+\mathrm{SD}$ & \\
\hline Weight $(\mathrm{Kg})$ & 45.0000 & 4.7889 & 52.5455 & 7.2679 & $0.003^{* *}$ \\
\hline Height $(\mathrm{cm})$ & 134.0250 & 4.2246 & 139.4636 & 8.9769 & 0.084 \\
\hline $\mathrm{BMI}\left(\mathrm{Kg} / \mathrm{cm}^{2}\right)$ & 24.9648 & 1.0822 & 26.9341 & 1.8743 & $0.007^{* *}$ \\
\hline Waist C $(\mathrm{cm})$ & 76.3250 & 4.6426 & 76.9273 & 4.0655 & 0.731 \\
\hline $\mathrm{Hip}$ C $(\mathrm{cm})$ & 86.8500 & 4.9280 & 91.5091 & 6.0855 & $0.038^{*}$ \\
\hline $\begin{array}{l}\text { Waist/Hip ratio } \\
\text { Skinfold }(\mathrm{mm})\end{array}$ & .8787 & 0.01 & .8423 & 0.04 & $0.023^{*}$ \\
\hline Triceps & 26.2500 & 4.973 & 26.0091 & 5.489 & 0.907 \\
\hline Biceps & 20.0000 & 2.633 & 18.0000 & 5.300 & 0.267 \\
\hline Subscaular & 23.2500 & 1.5275 & 27.0727 & 5.4172 & $0.043^{*}$ \\
\hline Suprailiac & 25.7500 & 3.8902 & 26.5818 & 5.7803 & 0.658 \\
\hline Abdominal & 27.8500 & 4.3534 & 25.0727 & 6.9950 & 0.214 \\
\hline Peripheral fat & 46.2500 & 6.6282 & 44.0091 & 7.0456 & 0.408 \\
\hline Central fat & 76.8500 & 7.4596 & 78.7273 & 16.5460 & 0.730 \\
\hline \multicolumn{6}{|l|}{ Laboratory data } \\
\hline Fasting Bl. sugar & 104.7500 & 5.904 & 90.3818 & 28.718 & 0.131 \\
\hline TG & 110.6872 & 17.4436 & 138.6564 & 37.8269 & $0.039^{*}$ \\
\hline TC & 201.3075 & 40.5578 & 161.4460 & 40.9913 & $0.019^{*}$ \\
\hline HDL-C & 30.1595 & 12.8451 & 44.8783 & 19.2251 & $0.027^{*}$ \\
\hline LDL-C & 148.8295 & 41.1653 & 86.0085 & 48.9964 & $0.002^{* *}$ \\
\hline
\end{tabular}

done using t-test as all the parameter are normally distributed.

measurements and all lipid profile parameters. Girls had significantly higher mean values of body weight, BMI, hip circumference, sub scapular skinfold thickness, high triglycrides and HDL-C within normal range $(p<0.05)$ than boys, while waist/ hip ratio, high LDL-C and total cholesterol were significantly higher in boys. While in group (II) aged 11-13 years \pm 6 months (Table 2), girls

Table 2: Anthropometric characteristics and laboratory data of the group (II) by sex (Age $11-13 \pm 6$ months).

\begin{tabular}{|c|c|c|c|c|c|}
\hline \multirow[t]{2}{*}{ Parameters } & \multicolumn{2}{|c|}{$\begin{array}{c}\text { Boys } \\
(N=29)\end{array}$} & \multicolumn{2}{|c|}{$\begin{array}{c}\text { Girls } \\
(N=33)\end{array}$} & \multirow[t]{2}{*}{$\mathrm{p}$} \\
\hline & Mean & $+S D$ & Mean & $+\mathrm{SD}$ & \\
\hline Weight $(\mathrm{Kg}) \dagger$ & 67.2241 & 11.4963 & 74.2455 & 9.3644 & $0.010^{* \star}$ \\
\hline Height $(\mathrm{cm}) \dagger$ & 150.1552 & 8.6072 & 154.3485 & 6.7412 & $0.036^{*}$ \\
\hline BMI $\left(\mathrm{Kg} / \mathrm{cm}^{2}\right) \dagger$ & 29.5513 & 2.3308 & 31.0713 & 2.6301 & $0.020^{*}$ \\
\hline Waist C $(\mathrm{cm}) \ddagger$ & 87.5172 & 11.7035 & 95.0909 & 23.9576 & 0.927 \\
\hline $\mathrm{Hip} \mathrm{C}(\mathrm{cm}) \dagger$ & 101.1724 & 8.3967 & 102.4545 & 10.3478 & 0.597 \\
\hline $\begin{array}{l}\text { Waist/Hip ratio } \neq \\
\text { Skinfold }(\mathrm{mm})\end{array}$ & .8617 & 0.05 & .9556 & .3637 & 0.076 \\
\hline Triceps $†$ & 27.1071 & 4.969 & 26.7839 & 6.267 & 0.828 \\
\hline Biceps $†$ & 19.9071 & 6.349 & 17.4710 & 5.772 & 0.128 \\
\hline Subscaulart & 26.0214 & 5.6046 & 27.6065 & 5.3969 & 0.273 \\
\hline Suprailiact & 25.3500 & 5.5347 & 26.8097 & 6.8729 & 0.376 \\
\hline Abdominal $\dagger$ & 29.5500 & 5.9700 & 25.4677 & 6.1576 & $0.012^{*}$ \\
\hline Peripheral fat $†$ & 47.0143 & 10.6482 & 44.2548 & 10.8239 & 0.329 \\
\hline Central fat $†$ & 80.9214 & 14.2400 & 79.8839 & 14.7713 & 0.785 \\
\hline \multicolumn{6}{|l|}{ Laboratory data } \\
\hline Fasting Bl. sugart & 98.6621 & 15.888 & 100.5455 & 11.437 & 0.599 \\
\hline TG $†$ & 136.4539 & 34.2146 & 144.6530 & 44.8485 & 0.432 \\
\hline $\mathrm{TC}+$ & 179.9251 & 63.5769 & 189.5879 & 58.5681 & 0.539 \\
\hline $\mathrm{HDL}-\mathrm{C} \dagger$ & 41.7939 & 11.8665 & 34.8089 & 21.9199 & 0.125 \\
\hline LDL-C† & 112.5897 & 61.9323 & 129.5482 & 56.0266 & 0.266 \\
\hline
\end{tabular}

N.B.: ${ }^{*} p<0.05=$ Significant differences. $\uparrow$ Comparisons done using t-testt as the parameter is normally distributed. $\neq$ Comparisons done using Mann-Whitney Test as the parameter is not normally distributed.

had significant gender differences for body weight, height and BMI. While boys had significantly higher values of abdominal skin fold thickness than girls. Group III aged 14-16 years \pm 6 months (Table 3 ) showed significant 
Table 3: Anthropometric characteristics and laboratory data of the group (III) by sex (Age 14-16 \pm 6 months).

\begin{tabular}{|c|c|c|c|c|c|}
\hline \multirow[t]{2}{*}{ Parameters } & \multicolumn{2}{|c|}{$\begin{array}{c}\text { Boys } \\
(N=25)\end{array}$} & \multicolumn{2}{|c|}{$\begin{array}{c}\text { Girls } \\
(N=25)\end{array}$} & \multirow[t]{2}{*}{$p$} \\
\hline & Mean & $+\mathrm{SD}$ & Mean & $+\mathrm{SD}$ & \\
\hline Weight $(\mathrm{Kg}) \dagger$ & 89.9680 & 12.1175 & 88.1560 & 10.5521 & 0.575 \\
\hline Height $(\mathrm{cm}) \dagger$ & 166.5640 & 9.4539 & 160.4400 & 8.3530 & $0.019^{*}$ \\
\hline BMI $\left(\mathrm{Kg} / \mathrm{cm}^{2}\right) \dagger$ & 32.3639 & 2.8282 & 34.2464 & 3.3732 & $0.038^{*}$ \\
\hline Waist C $(\mathrm{cm}) \ddagger$ & 104.9200 & 14.9033 & 91.6476 & 7.0508 & $0.000^{* *}$ \\
\hline $\mathrm{Hip} \mathrm{C}(\mathrm{cm}) \dagger$ & 114.6120 & 9.4225 & 111.6667 & 9.2256 & 0.292 \\
\hline $\begin{array}{l}\text { Waist/Hip ratioł } \\
\text { Skinfold }(\mathrm{mm})\end{array}$ & .9192 & .1421 & .8231 & 0.05 & $0.000^{* *}$ \\
\hline Triceps $†$ & 26.9476 & 6.376 & 27.3480 & 8.155 & 0.856 \\
\hline Biceps $†$ & 21.2429 & 8.300 & 22.0880 & 7.470 & 0.718 \\
\hline Subscaular† & 33.2524 & 6.4419 & 30.5800 & 8.3438 & 0.238 \\
\hline Suprailiac $\dagger$ & 29.9000 & 10.1188 & 23.1200 & 7.6666 & $0.016^{*}$ \\
\hline Abdominal† & 36.8333 & 7.5565 & 24.4800 & 7.6369 & $0.000^{\star *}$ \\
\hline Peripheral fat $†$ & 48.1905 & 12.8845 & 49.4360 & 14.4761 & 0.761 \\
\hline Central fat $†$ & 99.9857 & 19.1086 & 78.1800 & 21.2930 & $0.001^{\text {**}}$ \\
\hline \multicolumn{6}{|l|}{ Laboratory data } \\
\hline FBS & 94.7160 & 15.285 & 83.4160 & 12.925 & $0.007^{* *}$ \\
\hline TG $\dagger$ & 132.1809 & 71.4628 & 143.4132 & 69.5784 & 0.576 \\
\hline TC† & 173.8990 & 56.0588 & 174.7620 & 61.3258 & 0.959 \\
\hline $\mathrm{HDL}-\mathrm{C}+$ & 43.7640 & 17.3949 & 59.1586 & 32.7036 & $0.044^{*}$ \\
\hline LDL-C† & 102.0242 & 58.1636 & 85.8683 & 65.6661 & 0.366 \\
\hline
\end{tabular}

N.B.: ${ }^{*} \mathrm{p}<0.05=$ Significant differences. ${ }^{* \star} \mathrm{p}<0.01=$ highly significant differences. $†$ Comparisons done using t-testt as the parameter is normally distributed. ‡Comparisons done using MannWhitney Test as the parameter is not normally distributed.

gender differences for body height, waist circumference, waist/ hip ratio, suprailiac and abdominal skin fold thicknesses, central adiposity and fasting blood sugar where boys had the higher values. While girls had significant higher value of BMI and HDL-C. It was obvious that boys had significant tendency for increase in the anthropometric parameters indicating central obesity with increase in age, inspite of having high LDL-C and low HDL-C in young age only. While girls had significant higher values of BMI in the three age groups.

Correlation of fasting blood sugar and lipid profiles and the anthropometric measurements for all groups by gender are presented in tables 4 and 5 . For boys (Table 4), fasting blood sugar showed significant strong positive correlations with weight, BMI, waist circumference, hip circumference, triceps and subscapular skinfold thicknesses in group (I) aged 8-10 years. While in both groups II and III, fasting blood sugar had significant negative correlations with biceps and suprailiac skinfold thickness. Moreover, fasting blood sugar had significant negative correlations with abdominal skinfold thickness in second group, and with hip circumference, peripheral and central fat in the third group. Triglycerides had significant negative correlations with subscapular skinfold thickness in the first group, and with biceps skinfold thickness in the third group. Inspite of that, it had significant positive correlations with triceps, subscapular, suprailiac skinfold thickness and central fat in second group. Total cholesterol had significant negative correlations with waist/hip ratio and subscapular skinfold thickness in the first group, and with triceps and suprailiac skinfold thickness in the second group. HDL-C had significant negative correlations with biceps and abdominal skinfold thickness and peripheral and central fat in both first and second groups only. While LDL-C had significant negative correlations with subscapular skinfold thickness in first group, and positive one in second group.

For girls (Table 5), in group I (8-10 years), triglyceride had significant positive correlation with waist circumference and subscapular skinfold thickness. For group (II) aged 11-13 years \pm 6 months, none of the parameters under study showed any significant correlation except HDL-C which had significant negative correlations with subscapular skinfold thickness. Group III aged 14-16 years \pm 6 months, fasting blood sugar only had significant positive correlation with BMI.

It was obvious that for boys, fasting blood sugar correlated with most of the anthropometric measurements

Table 4: Correlations of anthropometric indices with laboratory data of obese boys.

\begin{tabular}{|c|c|c|c|c|c|c|c|c|c|c|c|c|c|c|c|}
\hline & \multicolumn{5}{|c|}{$\begin{array}{c}\text { Group I } \\
8-10 \text { y }(n=16)\end{array}$} & \multicolumn{5}{|c|}{$\begin{array}{c}\text { Group II } \\
11-13 \text { y }(n=29)\end{array}$} & \multicolumn{5}{|c|}{$\begin{array}{c}\text { Group III } \\
14-16 y(n=25) \\
\end{array}$} \\
\hline & FBS & TG & $\mathrm{TC}$ & HDL-C & LDL-C & FBS & TG & $\mathrm{TC}$ & HDL-C & LDL-C & FBS & TG & $\mathrm{TC}$ & $\mathrm{HDL}-\mathrm{C}$ & LDL-C \\
\hline \multicolumn{16}{|l|}{ Anthropometry } \\
\hline Weight $(\mathrm{Kg})+$ & $0.783^{* *}$ & -.061 & -.055 & .169 & -.093 & .269 & .164 & -.012 & -.086 & .001 & -.239 & .036 & -.089 & -.117 & .020 \\
\hline BMI $\left(K g / \mathrm{cm}^{2}\right) \dagger$ & $0.760^{\star *}$ & -.034 & -.020 & .151 & -.059 & .023 & .122 & -.237 & -.241 & -.207 & -.342 & -.130 & -.333 & .057 & -.269 \\
\hline Waist C $(\mathrm{cm}) \pm$ & $0.893^{* *}$ & -.211 & -399 & $.546^{*}$ & $-.538^{*}$ & .124 & .040 & -.002 & -.116 & .060 & -.136 & -.336 & -.342 & -.007 & -220 \\
\hline $\mathrm{Hip} \mathrm{C}(\mathrm{cm}) \dagger$ & $0.904^{* *}$ & -.215 & -.305 & .349 & -.385 & .094 & .349 & .120 & $-.414^{*}$ & .188 & $-.507^{* *}$ & -.327 & -.248 & .020 & -.289 \\
\hline Waist/Hip ratioł & 0.251 & -.085 & $-.500^{*}$ & $.881^{* *}$ & $-.755^{\star *}$ & .320 & .114 & .204 & -.078 & .239 & .313 & .198 & -.033 & -.061 & .101 \\
\hline \multicolumn{16}{|l|}{ Skinfold $(\mathrm{mm})$} \\
\hline Triceps $\dagger$ & $0.556^{*}$ & .050 & .208 & -.166 & .254 & -.128 & $.522^{* *}$ & $.417^{*}$ & -.203 & $.410^{*}$ & -.399 & .032 & .067 & .055 & .169 \\
\hline Biceps $t$ & 0.017 & -.288 & .144 & $-.949^{* *}$ & .459 & $-.455^{*}$ & .231 & .189 & $-.495^{* *}$ & .275 & $-.601^{* *}$ & $-.457^{*}$ & .232 & .244 & .142 \\
\hline Subscaular† & $0.983^{* *}$ & $-.728^{* *}$ & $-.790^{* *}$ & .175 & $-.764^{* *}$ & .172 & $.384^{*}$ & .338 & -.335 & $.382^{*}$ & -.411 & -.286 & -.023 & .004 & .071 \\
\hline Suprailiact & 0.357 & .294 & .440 & -127 & .449 & $-.428^{*}$ & $.563^{* *}$ & $.418^{*}$ & -.346 & $.431^{*}$ & $-.463^{*}$ & -.060 & .056 & .114 & -.003 \\
\hline Abdominalt & 0.040 & -.234 & .194 & $-.919^{* *}$ & .494 & $-.550^{* *}$ & .379 & -.061 & $-.680^{* *}$ & .010 & -.253 & -.292 & .068 & .007 & .151 \\
\hline Peripheral fat† & 0.424 & -.077 & .213 & $-.501^{*}$ & .373 & -.331 & .380 & .306 & $-.389^{*}$ & .356 & $-.584^{* *}$ & -.278 & .182 & .184 & .175 \\
\hline Central fatt & 0.411 & -.132 & .181 & $-.567^{*}$ & .366 & -.329 & $.515^{\star *}$ & .264 & $-.534^{* *}$ & .322 & $-.484^{*}$ & -.244 & .049 & .064 & .082 \\
\hline
\end{tabular}

N.B. ${ }^{*} p<0.05=$ Significant differences. ${ }^{*} p<0.01=$ highly significant differences. $†$ Pearson s correlation was used as the parameter is normally distributed. $\neq$ Spearman correlation was used as the parameter is not normally distributed. 
Table 5: Correlations of anthropometric indices with laboratory data of obese girls.

\begin{tabular}{|c|c|c|c|c|c|c|c|c|c|c|c|c|c|c|c|}
\hline & \multicolumn{5}{|c|}{$\begin{array}{c}\text { Group I } \\
8-10 \text { y }(n=11)\end{array}$} & \multicolumn{5}{|c|}{$\begin{array}{c}\text { Group II } \\
11-13 \text { y }(n=33)\end{array}$} & \multicolumn{5}{|c|}{$\begin{array}{c}\text { Group III } \\
14-16 \text { y }(n=25)\end{array}$} \\
\hline & FBS & TG & TंC & HDL-C & LDL-C & FBS & TG & TC & HDL-C & LDL-C & FBS & TG & $\mathrm{TC}$ & HDL-C & LDL-C \\
\hline $\begin{array}{r}\text { Anthropometry } \\
\text { Weight }(\mathrm{Kg}) \dagger\end{array}$ & .173 & .253 & -.157 & -.072 & -.197 & .125 & .291 & -.252 & -.158 & -.207 & .091 & .162 & .188 & .147 & .041 \\
\hline $\begin{array}{l}\text { BMI }\left(\mathrm{Kg} / \mathrm{cm}^{2}\right) \dagger \\
\text { Waist C }(\mathrm{cm}) \ddagger \\
\text { Hip C }(\mathrm{cm}) \dagger \\
\text { Waist/Hip ratio } \neq\end{array}$ & $\begin{array}{l}.250 \\
.014 \\
-.140 \\
.181\end{array}$ & $\begin{array}{l}.186 \\
.625^{\star} \\
.528 \\
-.012\end{array}$ & $\begin{array}{l}-.051 \\
-.369 \\
-.460 \\
.182\end{array}$ & $\begin{array}{l}-.428 \\
-.287 \\
.111 \\
-.403\end{array}$ & $\begin{array}{l}.118 \\
-.492 \\
-.601 \\
.290\end{array}$ & $\begin{array}{l}.333 \\
-.123 \\
.152 \\
-.303\end{array}$ & $\begin{array}{l}.073 \\
.008 \\
.115 \\
-.175\end{array}$ & $\begin{array}{l}.027 \\
-.110 \\
-.152 \\
.038\end{array}$ & $\begin{array}{l}.136 \\
-.275 \\
.072 \\
-.132\end{array}$ & $\begin{array}{l}-.020 \\
-.012 \\
-.177 \\
.140\end{array}$ & $\begin{array}{l}.502^{\star} \\
-.094 \\
.300 \\
-.459\end{array}$ & $\begin{array}{l}-.027 \\
-.109 \\
-.204 \\
-.088\end{array}$ & $\begin{array}{l}.122 \\
.299 \\
.145 \\
.163\end{array}$ & $\begin{array}{l}-.026 \\
-.027 \\
.039 \\
-.188\end{array}$ & $\begin{array}{l}.108 \\
.283 \\
.114 \\
.219\end{array}$ \\
\hline $\begin{array}{c}\text { skinfold }(\mathrm{mm}) \\
\text { Triceps } † \\
\text { Biceps } † \\
\text { Subscaular† } \\
\text { Suprailiac† }\end{array}$ & $\begin{array}{l}.090 \\
.309 \\
.210 \\
-.045\end{array}$ & $\begin{array}{l}-.132 \\
.404 \\
.624^{*} \\
.514\end{array}$ & $\begin{array}{r}.145 \\
.233 \\
.092 \\
-.103\end{array}$ & $\begin{array}{l}-.177 \\
-.230 \\
.116 \\
.178\end{array}$ & $\begin{array}{l}.345 \\
.076 \\
-.082 \\
-.199\end{array}$ & $\begin{array}{l}.168 \\
.089 \\
-.316 \\
-.221\end{array}$ & $\begin{array}{l}-.008 \\
-.325 \\
.072 \\
-.149\end{array}$ & $\begin{array}{l}.162 \\
-.012 \\
-.338 \\
-.238\end{array}$ & $\begin{array}{c}.025 \\
.025 \\
-.392^{*} \\
.039\end{array}$ & $\begin{array}{l}.184 \\
.007 \\
-.166 \\
-.193\end{array}$ & $\begin{array}{l}-.052 \\
-.050 \\
.070 \\
-.072\end{array}$ & $\begin{array}{l}.238 \\
.194 \\
.185 \\
.280\end{array}$ & $\begin{array}{l}-.207 \\
-.009 \\
.022 \\
-.039\end{array}$ & $\begin{array}{l}-.256 \\
-.149 \\
-.043 \\
.016\end{array}$ & $\begin{array}{r}-.124 \\
.020 \\
.002 \\
-.124\end{array}$ \\
\hline $\begin{array}{l}\text { Abdominalt } \\
\text { 'eripheral fat† } \\
\text { Jentral fatt }\end{array}$ & $\begin{array}{l}-.250 \\
.303 \\
-.053\end{array}$ & $\begin{array}{l}.217 \\
.201 \\
.476\end{array}$ & $\begin{array}{l}-.392 \\
.288 \\
-.171\end{array}$ & $\begin{array}{l}-.100 \\
-.240 \\
.065\end{array}$ & $\begin{array}{l}-.284 \\
.281 \\
-.205\end{array}$ & $\begin{array}{l}-.032 \\
.145 \\
-.232\end{array}$ & $\begin{array}{l}0.010 \\
-.178 \\
-.039\end{array}$ & $\begin{array}{l}-.075 \\
.087 \\
-.265\end{array}$ & $\begin{array}{l}-.167 \\
.028 \\
-.196\end{array}$ & $\begin{array}{r}.043 \\
.110 \\
-.135\end{array}$ & $\begin{array}{l}-.005 \\
-.055 \\
0.000\end{array}$ & $\begin{array}{l}.376 \\
.234 \\
.308\end{array}$ & $\begin{array}{l}-.062 \\
-.121 \\
-.027\end{array}$ & $\begin{array}{l}-.020 \\
-.221 \\
-.018\end{array}$ & $\begin{array}{l}-.153 \\
-.059 \\
-.099\end{array}$ \\
\hline
\end{tabular}

N.B.: ${ }^{\star} p<0.05=$ Significant differences. ${ }^{*}{ }^{*}<0.01=$ highly significant differences. $†$ Pearson $s$ correlation was used as the parameter is normally distributed. $\neq$ Spearman correlation was used N.B.:* $<<0.05=$ Significant differences. ${ }^{\star} \mathrm{p}<0.01$
as the parameter is not normally distributed.

in young age, and with skinfold thickness in the three age groups. While lipid profile correlated with skinfold thickness particularly at subscapular area in the age groups I and II (8 -13 years). For girls, none of the parameters under study showed any significant correlation except subscapular skinfold thickness which had significant correlations with lipid profile in age groups I and II (8 -13 years).

\section{Discussion}

Childhood is an important period in individual's life and appears to be a critical period for the development of obesity. Although the fundamental causes of epidemic obesity are sedentary life style and high calorie energy dense diet, yet, obesity participates in the pathway that increases the risk for developing cardio-metabolic diseases. However, obesity is associated with a broad range of health issues ranging from specific diseases (type II diabetes and hypertension) to impaired quality of life [18].

As far as we knew, most adipose tissue ( $85 \%$ of total adipose tissue mass) is located under the skin (subcutaneous fat), and smaller ( 15\%) is located within the abdomen (intra-abdominal fat) in lean and obese persons [19]. The relative contribution of intra-abdominal fat mass to total body fat is influenced by sex, age, race, ethnicity, physical activity, and total adiposity [20].

The current results revealed prominent interactions of age and sex on the development of obesity, as reflected by the significant higher values of abdominal skin fold thicknesses for boys in the age groups I, II ( 11 -16 years old) and subsequently, in central obesity in adolescence (14-16 years old). Inspite of existing of significant high LDL-C and low HDL-C in young age only. Boys had higher values of abdominal skinfold thickness in the 3 age groups, which was significant only in older two age groups (age from 11 to 16 years), and in central adiposity, waist circumference, waist/hip ratio and subscapular skinfold thickness in adolescents aged 14 -16 years, and also high LDL-C and low HDL-C in young age. While girls had significant higher values of BMI, high triglycrides levl and high HDLC (significant in group I only) in the three age groups. This is in agreement with results of many authors, and it may be attributed to the separate threshold of sex specific difference in pattern of fat distribution throughout the body, as well as, to the stage of sexual maturation [6, 19-23]. Hashemipour et al., found that the prevalence of high LDL-C and low HDL-C was higher in boys than in girls aged 6-11 years [4].

The term "visceral fat" is commonly used to describe intra-abdominal fat and it includes both intraperitoneal fat (mesenteric and omental fat), which drains directly into portal circulation, and retroperitoneal fat, which drains into the systemic circulation. The relation between visceral fat and plasma lipid-lipoprotein concentration may be explained by the peculiar feature of intra-abdominal adipocytes. This fat depot has a high lipolytic rate resulting in an increased fatty acid flux draining into the portal circulation. The consequent enhanced expose of the liver to increased fatty acids and insulin may be important in the pathogenesis of increased TG in central obesity. Furthermore, the exposure of peripheral tissue to high concentration of fatty acids may lead to decreased tissue sensitivity to insulin [19]. The current study addresses the link between anthropometric 
parameters especially central (visceral) fat and cardio metabolic disease risk in obese pediatric population.

Presence of childhood obesity determined a greater chance of finding high level of LDL-C and triglyceride (TG). Our findings are consistent with many previous studies $[6,7,18,19,24]$, where the anthropometric variables exhibited diagnostic capacity for cardio metabolic risk factors for younger boys aged from 8-16 years provided by the presence of significant association where fasting blood sugar correlated with most of the anthropometric measurements in young age, and with skinfold thickness in the three age groups. While lipid profile correlated with skinfold thickness particularly at subscapular area in the age groups I and II (8 -13 years).For girls, none of the parameters under study showed any significant correlation except subscapular skinfold thickness which had significant correlations with lipid profile in age groups I and II (8 -13 years).

For adolescents, there is insignificant correlation between any one of the lipid profile and most of the anthropometric parameters in either gender. This finding came in agree with that of Hashemipour et al.,[4] where they concluded that no single anthropometric index was found to be associated with risk factors in different sex and age groups. However, study of Kelishadi et al., 2007, [25] in Iran, documented that BMI, WC and WHR were the most appropriate indices in predicting lipid profile among youths.

In the current study, WHR was a good predictor of lipid profile (cardio-metabolic risk factors) for young boys (8-10 years old) and not in adolescents (11- 16 years old); this might be because of the physiologic changes in fat deposition during growth and subsequent puberty. Our finding is consistent with a previous study which determined the diagnostic accuracy for detecting excess body fatness, measured by different anthropometric indices among Swedish adolescents, using ROC analyses. In this study significant correlation was observed between HDL-C and anthropometric indexes. Similar studies among children have reported significant correlation for such variables [26, 27].

Some researchers have suggested that defining obesity by BMI has many disadvantages such as a lack of consensus on the cutoff for distinguishing overweight and obesity, and not presenting body fat distribution [1, 28]. However, we found that BMI had good correlation with fasting blood sugar and lipid profile in younger boys aged from 8-10 years.
These findings contradict those of Margolis et al., [29] who found high correlation between females' lipid level and body mass index, which may be attributed to the differences of age and nutritional status in the respective population.

This study has concluded that fat distribution among obese children; using anthropometric assessment

in form of skinfold thickness particularly subscapular; is important indicator for cardio-metabolic complications in both sexes. Young obese boys are more liable to cardiometabolic complications. This young age is critical and need to give more attention to avoid obesity complications in adult age.

\section{References}

1. Sweeting HN. Measurement and definitions of obesity in childhood and adolescence: a field guide for the uninitiated. Nutr J. 2007;6:32.

2. Mirzazadeh A., Sadeghirad B, Haghdoost AA, Bahrein F, Rezazadeh Kermani M. The Prevalence of Obesity in Iran in Recent Decade; a Systematic Review and Meta-Analysis Study. Iranian J Publ Health. 2009;38(3)1-11.

3. Kanniyappan D, Kalidhas P, Aruna RM. Age, gender related prevalence of cardiovascular risk factors in overweight and obese south Indian adults. Int J Biol Med Res. 2011; 2(2): 13522 (Desigamani et. al., 2011).

4. Mahin $H$, Soghrati $M$, Malekahmadi $M$, Soghrati $M$, Mirmoghtadaei P, Poursafa P, Kelishadi R. Association of Anthropometric Indexes and Cardiometabolic Risk Factors Among Obese Children. ARYAAtherosclerosis Journal. 2009; 5(1):39-48.

5. Wang Z, Hoy WE. Waist Circumference, Body Mass Index, Hip Circumference and Waist-To-Hip Ratio as Predictors of Cardiovascular Disease in Aboriginal People. Eur J Clin Nutr. 2004;58:888-893.

6. Hirschler V, Aranda C, Calcagno Mde L, Maccalini G, Jadzinsky M. Can waist circumference identify children with the metabolic syndrome? Arch Pediatr Adolesc Med. 2005; 159(8):740-4.

7. Kamath A, Nandini M, Kedilaya HP, D'Souza V (2005): Relation of anthropometry to CVD risk factors in young obese women. Biomedical Research. 2005;16(2):137-141.

8. Chadha DS, Singh G, Kharbanda P, Vasdev V, Ganjoo RK. Anthropometric correlation of lipid profile in healthy aviators Ind J Aerospace Med. 2006;50(2):32-37.

9. Shahraki T, Shahraki M, Roudbari M. Waist circumference: a better index of fat location than WHR for predicting lipid profile 
in overweight/obese Iranian women. East Mediterr Health J. 2009;15(4):899-905.

10. Priscila KM, Hallage T, Gama MPR, Sasaki JE, Miculis CP, Buzzachera CF, da Silva SG. Association Between Lipid Profile and Adiposity in Women Over Age 60. Arq Bras Cardiol. 2007;89(3):147-153.

11. Lean MJ \& Han TS. Waist worries. Am J Clin Nutr. 2002;76(4):699-700.

12. Ghalli I, Salah N, Hussien F, Erfan M, El- Ruby M, Mazen I, Sabry M, Abd El-Razik M, Saad M, Hossney L, Ismaail S and Abd El-Dayem S et al: Egyptian growth curves 2002 for infants, children and adolescents. published in: Sartorio A, Buckler JMH and Marazzi N. Crescere nel mondo. Ferring publisher, 2008.

13. Hiernaux J, Tanner JM. Growth and physical studies. In: Human Biology: A guide to Field Methods. Eds. Weiner JS, Lourie SA, IBP. London, Blackwell Scientific Publications. Oxford. UK, 1969:315-340.

14. Allain CC, Poon LS, Chen CSG, Richmond W, Fu PC. Enzymatic determination of total serum cholesterol. Clin Chem. 1974;20:470-475.

15. Fossati P, Prencipe L. Serum triglycerides determination colorimetrically with an enzyme that produces hydrogen peroxide. Clin Chem. 1982;28:2077-2088.

16. Burstein M, Scholnick HR, Morfin R. Rapid method for the isolation of lipoproteins from human serum by precipitation with polyanions. J Lipid Res. 1970;11:583-595.

17. Friedewald WT, Levy RI, Fredrickson DS. Estimation of the concentration of low density lipoprotein in plasma, without use of the preparative ultracentrifuge. Clin Chem. 972;18:499502.

18. Freedman DS, Katzmarzyk PT, Dietz WH, Srinivasan SR, Berenson GS. The relation of BMI and skinfold thicknesses to risk factors among young and middle-aged adults: the Bogalusa Heart Study. Ann Hum Biol. 2010;37(6):726-37.

19. Samue K, Allison DB, Heymsfield SB, Kelley DE, Leibel $\mathrm{RL}$, Nonas C, Kahn R. Waist circumference and cardiometabolic risk: a consensus statement from Shaping America's Health: Association for Weight Management and Obesity Prevention; NAASO, The Obesity Society; the American Society for Nutrition; and the American Diabetes Association. Am J Clin Nutr. 2007;85:1197-202.

20. Nooyens ACJ, Koppes LLJ, Visscher TLS, Twisk JWR,
Kemper HCG, Schuit AJ, van Mechelen W, Jacob C Seidell JC. Adolescent skinfold thickness is a better predictor of high body fatness in adults than is body mass index: the Amsterdam Growth and Health Longitudinal Study. American Journal of Clinical Nutrition. 2007;85(6):1533-1539.

21. Ebbeling CB, Pawlak DB, Ludwig DS. Childhood obesity: public-health crisis, common sense cure. Lancet. 2002; 360(9331):473-82.

22. Young $\mathrm{JH}$, Parler $\mathrm{P}$, Bristol B, Klag MJ. The coming epidemic: hypertension in rural Kyrgyzstan, Central Asia. J Hum Hypertens. 2005;19:145-148.

23. Bassali R, Waller JL, Gower B, Allison J, Davis CL. Utility of waist circumference percentile for risk evaluation in obese children. International Journal of Pediatric Obesity. 2010;5(1):97-101.

24. Choi JW, Pai SH, Kim SK. Associations Between Total Body Fat and Serum Lipid Concentrations in Obese Human Adolescents. Annals of Clinical \& Laboratory Science. 2002;32:271-278.

25. Kelishadi R, Gheiratmand R, Ardalan G, Adeli K, Mehdi Gouya M, Mohammad Razaghi E, Majdzadeh R, Delavari A, Shariatinejad K, Motaghian M, Heshmat R, Heidarzadeh A, Barekati H, Sadat Mahmoud-Arabi M, Mehdi Riazi M; CASPIAN Study Group. Association of anthropometric indices with cardiovascular disease risk factors among children and adolescents: CASPIAN Study. Int J Cardiol. 2007;117(3):3408.

26. Kortelainen ML, Sarkioja T. Coronary atherosclerosis and myocardial hypertrophy in relation to body fat distribution in healthy women: an autopsy study on 33 violent deaths. Int J Obes Relat Metab Disord. 1997;21(1):43-49.

27. Sarni RS, de Souza FI, Schoeps DO, Catherino P, de Oliveira MC, Pessotti CF, et al. Relationship between waist circumference and nutritional status, lipid profile and blood pressure in low socioeconomic level pre-school children. Arq Bras Cardiol. 2006;87(2):153-8.

28. Selçuk CA. Body mass index, wasit-to-height ratio, cardiometabolic risk factors and diseases in a new obesity classification proposal. Editorial. The Open Obesity Journal. 2011;3:56-61.

29. Margolis CF, Sprecher DL, Simbartl LA. Campaigne BN. Male-female differences in the relationship between obesity and lipids/lipoproteins. Int J Obes Relat Metab Disord. 1996;20:784-790. 